\title{
Age at menopause and hormone replacement therapy as risk factors for head and neck and oesophageal cancer (Review)
}

\author{
CAROLINE E. McCARTHY ${ }^{1,2}$, JOHN K. FIELD ${ }^{1,2}$ and MICHAEL W. MARCUS ${ }^{1}$ \\ ${ }^{1}$ Roy Castle Lung Cancer Research Programme, Department of Molecular and Clinical Cancer Medicine, \\ The University of Liverpool Institute of Translational Medicine, Liverpool L7 8TX; \\ ${ }^{2}$ Department of Oral Medicine, Liverpool University Dental Hospital, Liverpool L69 3BX, UK
}

Received September 2, 2016; Accepted February 16, 2017

DOI: $10.3892 /$ or.2017.5867

\begin{abstract}
There were $\sim 986,000$ cases of head and neck cancer (HNC) and oesophageal cancer diagnosed worldwide in 2012. The incidence of these types of cancer is much higher in males than females, although this disparity decreases in the elderly population, suggesting a role for hormones as a risk factor. This systematic review investigates the potential role of female hormones [age at menopause and use of hormone replacement therapy (HRT)] as risk factors for HNC/oesophageal squamous cell carcinoma (SCC). The electronic databases MEDLINE, Web of Science, EMBASE and Cochrane were searched. Only studies with at least 50 cases of $\mathrm{HNC/oesophageal} \mathrm{SCC,} \mathrm{with}$ data on age at menopause, smoking, alcohol, age and socioeconomic status or educational attainment, were included. The Newcastle Ottawa Scale was used for assessing risk of bias. Eight studies met the inclusion criteria (5 oesophageal SCC, $2 \mathrm{HNC}$ and 1 combined oesophageal SCC and HNC). HRT was shown to reduce the risk of HNC (HR, 0.78; 95\% CI, 0.610.99 ) in one study. Our results showed that earlier age at menopause is a risk factor for oesophageal SCC, with women entering menopause at $<45$ years having double the risk of those entering menopause at age $>50$ years. Similar, but less striking, results were observed for HNC. HRT was found to reduce the risk of HNC/oesophageal SCC, but the evidence is inconclusive. We, therefore, recommend that consideration should be given to collecting data on reproductive factors and exposure to HRT, as routine practice, in future epidemiological and clinical studies of these cancers. The concept of oestrogen deficiency as a risk for HNC/oesophageal SCC deserves further exploration in future laboratory and clinical studies.
\end{abstract}

Correspondence to: Dr Michael W. Marcus, Roy Castle Lung Cancer Research Programme, Department of Molecular and Clinical Cancer Medicine, The University of Liverpool Institute of Translational Medicine, The William Duncan Building, 6 West Derby Street, Liverpool L7 8TX, UK

E-mail: m.w.marcus@liverpool.ac.uk

Key words: age at menopause, hormone replacement therapy, risk factors, head and neck cancer, oesophageal cancer, systematic review

\section{Contents}

1. Introduction

2. Methods

3. Search results

4. Hormone replacement therapy (HRT)

4. Age at menopause

5. Discussion

\section{Introduction}

There were $\sim 986,000$ cases of head and neck cancer (HNC), and oesophageal cancer diagnosed worldwide in 2012. In the same year, in the UK, there were 5,000 cases of HNC in males and 2,500 in females (1). In 2013, there were 8,784 cases of oesophageal cancer, with a male:female ratio of $2: 1$. This makes HNC and oesophageal cancer the 12th and 13th most common cancers in the UK (2). The ratio of male:female cases of HNC in persons aged 50-60 years is $\sim 3: 1$, however, the gender disparity is reduced in the elderly population, with a male female ratio of 1.5:1 in individuals over 80 years of age (3). For oesophageal squamous cell carcinoma (SCC) the male:female ratio is lower, at 1.1:1.

Understanding the differences between subgroups of the population is important to ensure that policy makers can make informed decisions regarding preventive measures. Smoking and alcohol are the major risk factors for HNC and oesophageal SCC; however, the differences in incidence between age categories cannot be solely attributed to differences in smoking and alcohol consumption (4). Hormones are known to play an important role in several types of cancers, such as breast, ovarian and uterine, endometrial, prostate, testis and thyroid cancers.

Studies have been carried out on hormone-related risk factors for squamous cancers, such as oesophageal, cervical and lung cancer (5). While hormone replacement therapy (HRT) is a known risk factor for certain cancers (e.g. breast cancer), a recent meta-analysis found that the use of HRT is protective against oesophageal SCC (6). Early-menopause has also been linked to an increased risk of oesophageal SCC [risk ratio (RR), 1.32 (95\% CI, 1.11-1.56)/5 years younger at menopause] (7). Another meta-analysis suggested decreased 
risk of lung cancer in never-smoker females who use HRT [OR, 0.86 (95\% CI, 0.75-0.99)]. This leads to the hypothesis that hormone levels may be a potential risk factor for squamous cancers. There is uncertainty surrounding the role of female hormones and the risk of head and neck oesophageal SCC. However, to the best of our knowledge, no systematic review has been conducted to address this uncertainty. The aim of this systematic review was to address the uncertainty surrounding the role of female hormones and the risk of head and neck and oesophageal SCC by evaluating two specific questions: i) is early menopause a risk factor for $\mathrm{HNC}$ or oesophageal SCC and ii) is HRT protective against $\mathrm{HNC}$ or oesophageal SCC?

\section{Methods}

In the present study, we combined head and neck, and oesophageal SCC due to their histology and strong similarities in their epidemiology and aetiology.

Search strategy. Electronic databases MEDLINE, Web of Science, EMBASE and Cochrane were searched up until February 11, 2016. Search strategies were developed using Medical Subject Heading (MeSH) words such as: head and neck neoplasms, head and neck and oesophageal cancer, esophageal neoplasms, HRT, female hormone, early menopause and text words related to hormones and $\mathrm{HNC}$ or (o)esophageal cancer. Only search terms describing the association were used. Reference lists were also extensively searched and relevant papers obtained.

Eligibility criteria. Randomized controlled trials (RCTs), controlled (non-randomised) clinical trials (CCTs) or cluster trials, prospective and retrospective comparative cohort studies, case-control or nested case-control studies, and cross-sectional studies, addressing the question of female hormones as a risk factor for HNC or oesophageal SCC, were considered. Studies were included when they: i) examined the general adult population (age $>18$ years), specifically patients with at least 50 cases of HNC/oesophageal SCC and healthy controls; ii) addressed the question of HRT or reproductive factors (menopause) and HNC/oesophageal SCC; iii) administered HRT as an intervention for prevention of cancer or being therapeutically taken due to symptoms of menopause; iv) collected data on age at menopause, smoking, alcohol, age and socioeconomic status or educational attainment; and v) reported odds ratios, risk ratios or incidence/prevalence of HNC or oesophageal SCC defined using the World Health Organisation (WHO) classification of diseases ICD-10 codes, C00-15 and C30-31.

Cohort studies were only eligible when follow-up time was at least 5 years; case series and case reports were excluded. Only English language articles published in peer-reviewed journals, from 1948 to 2016, were considered.

Data extraction. Titles and/or abstracts of studies retrieved using the search strategy and those from additional sources were independently screened by two review authors (C.M. and M.M.), to identify studies that potentially met the inclusion criteria outlined above. Studies combining HNC with oesophageal squamous cell cancers were considered, but data were extracted to separate HNC and oesophageal cancer where possible. Data were extracted in all forms (e.g. dichotomous, continuous) as reported in the included studies. The full texts of these potentially eligible studies were independently assessed for eligibility by C.M. and M.M. Any disagreement over the eligibility of particular studies was resolved through discussion with a third reviewer (J.K.F.).

Risk of bias. The risk of bias was assessed using the Newcastle Ottawa Scale which includes the following areas of evaluation of the risk of bias: selection of patients, comparability of groups in the study, methods for assessing outcomes, proof of exposure and appropriate follow-up. Studies were categorised as low, medium, high or unclear risk of bias, using a star-based scoring system. A maximum of 9 stars are available; the higher the number of stars the lower the risk of bias. For ease of interpretation, a score of 7 or greater is considered 'low-risk' of bias, 4-6 is 'medium-risk' and 3 or below is considered 'high-risk' of bias.

\section{Search results}

The search identified 13 potentially eligible studies following the review of titles and abstracts of studies identified from the initial search. One study considered HNC and oesophageal cancer separately, therefore was included in both arms of the review. Five papers were excluded, based on insufficient number of cases $(n=2)$, failure to report an effect estimate/confidence intervals $(\mathrm{n}=2)$ and lack of categorisation by histopathological subtype $(n=1)$. Eight studies met the inclusion criteria $(4,7-13)$. The literature search results and selection process are depicted in Fig. 1A and B.

The systematic review includes two cohort studies (one oesophageal/HNC and one oesophageal cancer only) $(7,8)$ with follow-up time of 7.5 and 9.1 years, respectively. Six case-control studies (4 oesophageal cancer and 2 HNC) (4,9-13) were also included. Studies covered the UK $(7,13)$, USA $(4,8)$, European continent $(9,14)$ and China $(11,12)$. The mean number of cases/study for the HNC articles was 214 (range 149-297). For oesophageal cancer, the average case number/study was 163 (range 56-578). A summary of the demographic data for each study is presented in Table I. A summary of the findings regarding use of HRT and risk of HNC/oesophageal SCC is shown in Table IIA. Table IIB summarises the findings regarding age at menopause.

\section{Hormone replacement therapy (HRT)}

$H N C$. Three papers (1 cohort study and 2 case-control studies) addressed the question of the use of HRT and incidence of HNC. Only the study by Freedman et al (8) was considered at 'low-risk' of bias, with a Newcastle Ottawa score of 7/9 stars. This was a cohort study conducted in the USA, using the NIH-AARP Diet and Health Cohort, of 125,887 women. Two hundred and ninety-seven cases of HNC were identified with mean follow-up of 7.5 years. The risk of $\mathrm{HNC}$ was $22 \%$ lower for ever-users of HRT (HR, 0.78; 95\% CI, 0.61-0.99); $44.1 \%$ of cases $(n=127)$ had ever-used HRT compared to $54.6 \%$ of controls $(n=106,934)$. Further analysis by hysterectomy status 
A

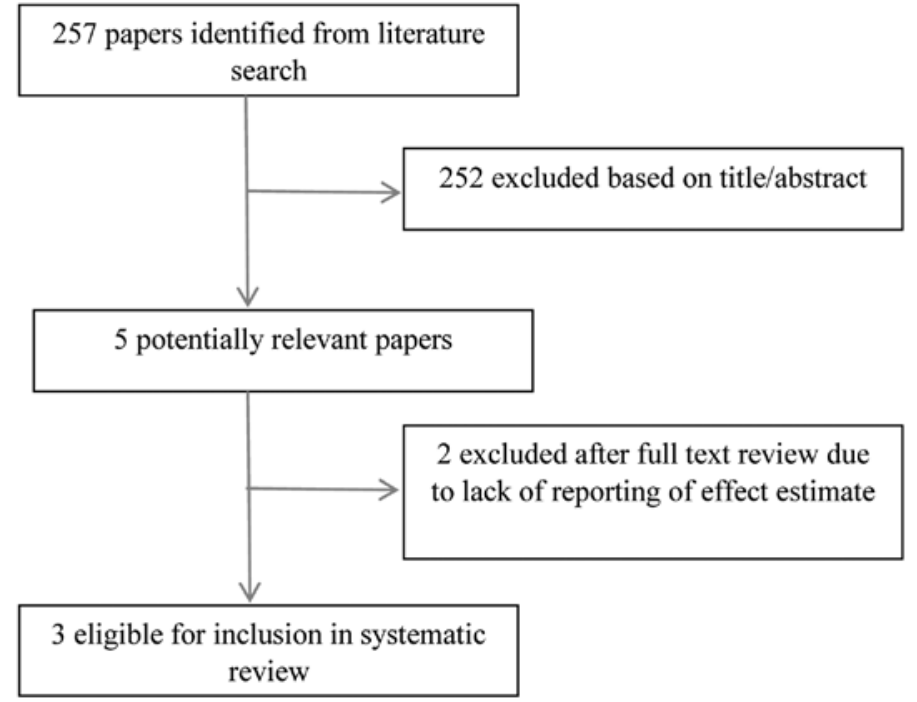

B

293 papers identified from literature search

278 excluded based on title/abstract

9 potentially relevant papers

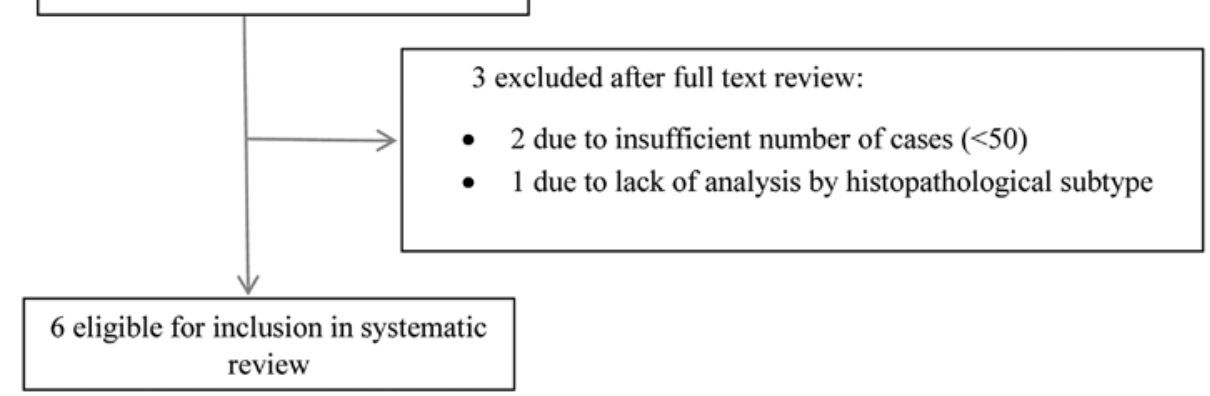

Figure 1. (A) Flow diagram of literature search results and selection process for head and neck cancer. (B) Flow diagram of literature search results and selection process for oesophageal cancer.

revealed that the risk reduction was greatest for women with an intact uterus who were current users of HRT for $>5$ years (HR, 0.23; 95\% CI, 0.09-0.57). Notably, use of HRT other than oestrogen-alone or oestrogen-progesterone therapy conferred a greater risk of $\mathrm{HNC}$ (HR, 2.31; 95\% CI, 1.15-4.65); however, this analysis was based on only 9 cases who used an alternative HRT. Two case-control studies were considered at medium/high risk of bias; they reported a non-significant reduction in the risk of HNC for ever-users of HRT $(4,9)$.

Oesophageal SCC. Four studies analysed HRT use and the risk of oesophageal SCC. Two were considered low-risk of bias $(8,13)$ and two were medium/high risk of bias $(10,11)$. Although, all studies reported an effect estimate of $<1$ for users of HRT, implying a protective effect, none of the results were statistically significant.

\section{Age at menopause}

$H N C$. Two studies $(8,9)$ assessed the link between age at menopause and risk of HNC. Bosetti et al (9) found a protective effect of later age at menopause ( $>50$ years), with an OR of 0.46 (95\% CI, 0.30-0.70). Freedman et al (8) found no significant effect on the risk of $\mathrm{HNC}$ with later age at menopause ( $>55$ years).

Oesophageal SCC. Four out of 5 studies reported a significant effect of age at menopause and risk of oesophageal SCC. The 
Table I. Demographic characteristics of the included studies.

\begin{tabular}{|c|c|c|c|c|c|}
\hline First author (year) (ref.) & Country & Study type & Cancer type & Participant demographics & Time period \\
\hline Freedman (2010) (8) & USA & Cohort & $\begin{array}{l}\mathrm{HNC} / \\
\text { oesophageal }\end{array}$ & $\begin{array}{l}\text { NIH-AARP Diet and Health Study cohort, } \\
\text { aged } 50-71 \text { years } \\
\text { (median follow-up } 7.5 \text { years) }\end{array}$ & $1995-2003$ \\
\hline Langevin (2011) (4) & USA & Case-control & $\mathrm{HNC}$ & $\begin{array}{l}\text { Cases of primary HNC and complaint-free } \\
\text { hospital controls at ENT Department, } \\
\text { University of Pittsburgh Medical Center }\end{array}$ & 2006-2010 \\
\hline Bosetti (2000) (9) & $\begin{array}{l}\text { Italy/ } \\
\text { Switzerland }\end{array}$ & Case-control & $\mathrm{HNC}$ & $\begin{array}{l}\text { Cases of histologically confirmed oral/ } \\
\text { pharyngeal cancer age }<75 \text { years attending } \\
\text { hospitals in Italy/Switzerland and hospital } \\
\text { controls with acute, non-neoplastic conditions }\end{array}$ & 1984-1997 \\
\hline Lindblad (2006) (13) & UK & $\begin{array}{l}\text { Nested } \\
\text { case-control }\end{array}$ & Oesophageal & $\begin{array}{l}\text { UK General Practice Research Database } \\
\text { (UK GPRD) cohort aged 50-84 years }\end{array}$ & 1994-2001 \\
\hline Gallus (2003) (14) & $\begin{array}{l}\text { Italy/ } \\
\text { Switzerland }\end{array}$ & Case-control & Oesophageal & $\begin{array}{l}\text { Cases aged }<79 \text { years with histologically } \\
\text { confirmed oesophageal SCC admitted to } \\
\text { study hospitals; hospital controls } \\
\text { admitted to the same hospitals } \\
\text { for acute, non-neoplastic conditions }\end{array}$ & 1984-1999 \\
\hline Yu (2011) (11) & China & Case-control & Oesophageal & $\begin{array}{l}\text { Cases of histopathologically confirmed } \\
\text { oesophageal SCC; hospital based } \\
\text { controls confirmed not to have } \\
\text { oesophageal cancer }\end{array}$ & $2008-2010$ \\
\hline Chen (2011) (12) & China & Case-control & Oesophageal & $\begin{array}{l}\text { Cases of newly diagnosed primary } \\
\text { oesophageal cancer; hospital controls } \\
\text { with no history of cancer }\end{array}$ & 2004-2010 \\
\hline Green (2012) (7) & UK & Cohort & Oesophageal & $\begin{array}{l}\text { Million Women Study cohort } \\
\text { (women aged 50-64 years) } \\
\text { with mean 9.1 years follow-up }\end{array}$ & 1996-2008 \\
\hline
\end{tabular}

HNC, head and neck cancer.

method of reporting varied. Gallus et al (10) reported an OR of $0.43(95 \% \mathrm{CI}, 0.22-0.83)$ for age at menopause of $>50$ years vs. menopause at age $<45$ years. Yu et al (11) reported an increased risk of oesophageal SCC for women entering menopause at $<45$ years $(\mathrm{OR}, 2.27 ; 95 \% \mathrm{CI}, 1.03-4.97)$ and for 45-49 years (OR, 2.16; 95\% CI, 1.14-4.78) compared to menopause at age $>50$ years. Green et al $(7)$ reported increased risk of oesophageal SCC for every 5 years younger a women was at the time of menopause (RR, 1.32; 95\% CI, 1.11-1.56). Although, Freedman et al (8) found no significant effect for individual age categories, they did observe a significant trend $(p=0.019)$ for a lower risk of oesophageal SCC with older age at menopause. Chen et al (12) observed no significant effect for age at menopause, although these authors classified older age at menopause as $>48$ years.

\section{Discussion}

This systematic review has considered evidence from 8 studies investigating the risk of head and neck cancer (HNC) or oesophageal squamous cell carcinoma (SCC) in relation to age at menopause and use of hormone replacement therapy (HRT); 5 studies investigated oesophageal SCC, 2 studies investigated HNC and 1 study included both cancers.

Early menopause. We found that earlier age at menopause is associated with a higher risk of oesophageal cancer, based on 4 studies with a total of 836 cases of oesophageal SCC.

Most women experience menopause between the ages of 45 and 55 years; the median age at menopause is 47.2 years, according to a prospective cohort study of over 5,000 women enrolled at the Royal College of GP's Oral Contraception study (15). Menopause is considered to be early in women aged $40-45$ years ( $~ 5 \%$ of women) and premature in women $<40$ years ( $\sim 1 \%$ of women) (16).

Early menopause is more frequent in women with certain genetic or autoimmune disorders, infections or a history of chemotherapy/radiotherapy or surgery to remove the ovaries (16).

Mean age of menopause for smokers is significantly lower than non-smokers (45.6 vs. 46.9 years) (15). Women with early natural menopause are more likely to be smokers, ever-users 
Table II. The use of hormone replacement therapy and risk of HNC/oesophageal SCC, and age at menopause and risk of HNC/oesophageal SCC.

A, The use of hormone replacement therapy and risk of HNC/oesophageal SCC

\begin{tabular}{|c|c|c|c|c|c|c|}
\hline Outcome of interest & First author (year) (ref.) & Type of study & $\begin{array}{l}\text { No. of } \\
\text { cases }\end{array}$ & $\begin{array}{c}\text { Relative effect } \\
\text { (OR or HR) }\end{array}$ & $95 \%$ CI & $\begin{array}{l}\text { Risk of Bias } \\
\text { (Newcastle } \\
\text { Ottawa Scale) }\end{array}$ \\
\hline $\mathrm{HNC}$ & Freedman (2010) (8) & Cohort & 297 & HR 0.78 & $0.61-0.99$ & Low (8/9) \\
\hline $\mathrm{HNC}$ & Langevin (2011) (4) & Case-control & 149 & OR 0.47 & $0.20-1.08$ & Medium (6/9) \\
\hline $\mathrm{HNC}$ & Bosetti (2000) (9) & Case-control & 195 & OR 0.88 & $0.45-1.72$ & Medium-high (4/9) \\
\hline Oesophageal SCC & Lindblad (2006) (13) & Nested case-control & 74 & OR 0.93 & $0.40-2.16$ & Low (9/9) \\
\hline Oesophageal SCC & Gallus (2001) (10) & Case-control & 114 & OR 0.32 & $0.09-1.13$ & Medium-high (4/9) \\
\hline Oesophageal SCC & Freedman (2010) (8) & Cohort & 56 & HR 0.74 & $0.42-1.26$ & Low (8/9) \\
\hline Oesophageal SCC & $\mathrm{Yu}(2011)(11)$ & Case-control & 88 & OR 0.94 & $0.53-1.70$ & Medium-high (4/9) \\
\hline
\end{tabular}

Bold text denotes a statistically significant result.

B, Age at menopause and risk of HNC/oesophageal SCC

\begin{tabular}{|c|c|c|c|c|c|c|c|}
\hline Outcome of interest & First author (year) (ref.) & Type of study & $\begin{array}{l}\text { No. of } \\
\text { cases }\end{array}$ & $\begin{array}{l}\text { Age } \\
\text { (years) }\end{array}$ & $\begin{array}{c}\text { Relative effect } \\
\text { (OR or HR) }\end{array}$ & $95 \% \mathrm{CI}$ & $\begin{array}{c}\text { Risk of Bias } \\
\text { (Newcastle } \\
\text { Ottawa Scale) }\end{array}$ \\
\hline $\mathrm{HNC}$ & Freedman (2010) (8) & Cohort & 297 & $>55$ & HR 0.92 & $0.50-1.71$ & Low (8/9) \\
\hline $\mathrm{HNC}$ & Bosetti (2000) (9) & Case-control & 195 & $>50$ & OR 0.46 & $0.30-0.70$ & Medium-high (4/9) \\
\hline Oesophageal SCC & Gallus (2001) (10) & Case-control & 114 & $>50$ & OR 0.43 & $0.22-0.83$ & Medium-high (4/9) \\
\hline Oesophageal SCC & Freedman (2010) (8) & Cohort & 56 & $\begin{array}{l}\text { Increasing } \\
\text { age }\end{array}$ & Ptrend $=0.019$ & & Low $(8 / 9)$ \\
\hline Oesophageal SCC & Yu (2011) (11) & Case-control & 88 & $<45$ & OR 2.27 & $1.03-4.97$ & Medium-high (4/9) \\
\hline Oesophageal SCC & Green (2012) (7) & Cohort & 578 & $\begin{array}{l}\text { Per } 5 \text { years } \\
\text { younger }\end{array}$ & RR 1.32 & 1.11-1.56 & Low (7/9) \\
\hline Oesophageal SCC & Chen (2011) (12) & Case-control & 68 & $>48$ & OR 0.94 & $0.31-2.85$ & Medium $(5 / 10)$ \\
\hline
\end{tabular}

HNC, head and neck cancer; SCC, squamous cell carcinoma; OR, odds ratio; HR, hazard ratio; CI, confidence interval; RR, risk ratio. Bold text denotes a statistically significant result.

of oral contraceptive medication, undergone tubal ligation, have at least one episode of endometriosis and are less likely to use HRT. No association with alcohol, BMI, physical activity or parity was reported (15). Diabetic women have also been found to be at risk of early menopause (OR, 2.76; $95 \%$ CI, 1.32-5.66) (17). In a pooled analysis of case-control studies, diabetes diagnosed at age $<50$ years conferred a greater risk of $\mathrm{HNC}(\mathrm{OR}, 1.37$; 95\% CI, 1.07-1.74) when analysing 6,448 cases of HNC and 13,747 controls (18), but no link with age at menopause was considered in the present study.

A recent systematic review and meta-analysis of oesophageal SCC and reproductive factors also found a protective effect for older age at menopause (SRR=0.70; 95\% CI, 0.51-0.95). The authors concluded that 'properly extending the time of menstruation for pre-menopausal women is a possible way to reduce the risk of oesophageal SCC' (19). A separate metaanalysis found that menopausal status was associated with higher risk of oesophageal SCC (RR, 1.66; 95\% CI, 1.12-2.48), but age at menopause was not significant for Caucasians. In Asians, older age at menopause was found to confer greater risk of oesophageal SCC (RR, 1.68; 95\% CI, 1.05-2.70) (6). This implies that ethnicity and genetic background may influence the role of female hormones in oesophageal carcinogenesis.

The effect of age at menopause has been explored in several other cancer types. A meta-analysis of 8 studies of gastric cancer and reproductive factors found a protective effect of longer years of fertility ( $R R=0.74 ; 95 \% \mathrm{CI}, 0.63-0.86)(20)$. Early menopause has also been found to be a risk factor for bladder cancer (meta-analysis including 7 studies: $R R=1.59$; 95\% CI, 1.31-1.92) (21). Two studies from Hungary, from the same unit, found that the mean age at menopause was significantly lower in females with oral cancer compared to controls (43.5 vs. 50.9 and 45.4 years vs. 51.3 years, respectively) $(22,23)$. These studies were excluded from this review due to the failure to provide accurate effect estimates with confidence intervals. Other recent meta-analyses found no significant effect of age at menopause in relation to risk of brain tumours (24), pancreatic (25) or thyroid cancer (26). A large cohort study found no association with age at menopause and risk of colorectal cancer (27). 
$H R T$. HRT is long-established in the management of the symptoms of the menopause and has also been shown to reduce the risk of osteoporotic fractures, cardiovascular disease, Alzheimers, depression, stroke and colon cancer. Approximately $30 \%$ of UK women used HRT in 20012002 (28). Following this, a large US-based trial (Women's Health Initiative) was prematurely halted due to concerns over the evidence of increased risk of breast cancer, coronary heart disease, stroke and pulmonary embolism among users of HRT (29). The UK-based Million Women Study (MWS) also reported increased risk of breast cancer with HRT in 2003 (30). Following media coverage of the results of these trials, use of HRT declined steadily in the UK for the next 3-4 years. In 2005 , only $10-11 \%$ of menopausal women were using HRT (28). Further reports of a slight increased risk of ovarian cancer for ever-users of HRT compounds enhanced concerns over the safety of HRT (31).

However, concerns have been raised by some authors surrounding the reporting of the WHI and MWS trial results, and the fact that little coverage was given to the evidence of the reduced incidence of osteoporotic fractures and colon cancer (32). Both trials recruited women aged over 50 years; therefore, the results cannot be applied to women who undergo premature menopause (33). HRT for women with premature menopause (primary ovarian insufficiency), prescribed up to the age of natural menopause ( $\sim 51$ years), is endorsed by the British Menopause Society and NICE guidelines. The NICE guidelines also recommend the development of a collaborative 'primary ovarian insufficiency' registry to allow data collection to clarify, among other factors, the long-term risk of cancers in this group (34).

Despite reports of an increased risk of breast and ovarian cancers in certain groups using HRT, there are many observational studies reporting a reduced risk of other cancers in HRT users. There are several reports of a reduced risk of colon cancer with HRT $(27,35,36)$. Green et al reported a relative risk reduction of $17 \%$ (RR, 0.83 ; 95\% CI, 0.79-0.88) in their metaanalysis of 30 studies of colorectal cancer and HRT (36). In a recent meta-analysis of oesophageal cancer and reproductive factors, the authors reported a $33 \%$ relative risk reduction with HRT use (SRR, 0.67; 95\% CI, 0.56-0.81) (19). Similar results were reported for reduced risk of gastric cancers (RR, 0.77; 95\% CI, 0.64-0.92) (20). Combined HRT (oestrogen and progesterone) has also been shown to reduce the risk of lung cancer, in a meta-analysis of 25 studies (RR, 0.83; 95\% CI, 0.76-0.91) (37). HRT has not been found to significantly reduce the risk of melanoma or pancreatic cancer $(25,38)$.

This systematic review did not find evidence of a significant risk reduction for oesophageal cancer among users of HRT, in contrast to the findings of the meta-analysis by Zhu et al (19). However, all effect estimates were $<1$ and the studies included contained only modest numbers of oesophageal cancer cases. The Freedman et al (8) cohort study included 297 cases of $\mathrm{HNC}$ and was the only study to report a significant relative risk reduction for $\mathrm{HNC}$ in users of $\mathrm{HRT}$ (HR, 0.78; 95\% CI, 0.61-0.99). Further large studies are needed to confirm the finding that HRT is protective against HNC.

Confounding factors must be considered. Users of HRT tend to be of higher socioeconomic status (SES) and have higher levels of education. Both of these factors may reduce the risk of HNC. To address this, only studies controlling for a measure of SES or education were eligible for inclusion in this review. Freedman et al (8) controlled for education, alcohol, BMI, tobacco smoking, physical activity and diet (fruit and vegetable intake), although residual confounding factors could still be relevant.

Role of oestrogen deficiency. Females have been found to have survival advantage for HNC, oesophageal, gastric and pancreatic cancer, as well as cancers at 11 other sites. For all cancers combined, women have a $5 \%$ lower risk of death than men; for $\mathrm{HNC}$, a $12 \%$ increased survival is reported (39). This finding is consistent across all European regions in the EUROCARE-4 cohort of 1.6 million population-based cancer cases (39). The advantage is most pronounced in younger women and declines with age, with a marked decline beyond the age of menopause. It is possible that female hormones play a part in this $12 \%$ improvement in survival.

Oestrogen is known to promote cancer in oestrogen-responsive tissues, such as breast, endometrium and cervix; however, evidence from mouse models suggests that oestrogen has an inhibitory role in oesophageal SCC growth (40). Oestrogen receptors have been found in oesophageal SCC tissue samples and HNCs (40-42). Oesophageal SCC cells with oestrogen receptors have been shown to be inhibited by oestrogen exposure and this may initiate apoptosis (43). If oestrogen is responsible for inhibiting the growth of some cancer cells, oestrogen deficiency could be considered a risk factor for certain cancers. A women who undergoes premature menopause has less oestrogen exposure over her lifetime and this may increase her risk of cancers such as oesophageal SCC. However, oestrogen appears to have both tumorigenic and antitumour properties, depending on the tissue and presence of oestrogen receptors. HNC cell lines, from males and females, have been found to contain oestrogen receptors, and laboratory studies appear to show that oestrogen promotes the growth of HNC cells $(44,45)$. Further high quality basic science studies are required to confirm the role of oestrogen in HNC and oesophageal SCC.

The studies included in this review were all assessed for risk of bias, using the Newcastle Ottawa Scale. Only 3 of the 8 studies included were low risk for bias. This does limit the significance of our findings and is an indication of the need for further, high quality, studies addressing the issue of female hormones and squamous cancers.

We only included studies that controlled for significant potential confounding factors, such as smoking and socioeconomic status, however, the risk of residual confounding factors remains. Smokers undergo menopause at a younger age than non-smokers. We recommend that future studies should control for this important confounder.

The rationale for combining HNC and oesophageal SCC is based on the strong similarities in their epidemiology and aetiology as mentioned earlier. We deliberately excluded oesophageal adenocarcinomas as these cancers have quite different aetiology. One of the studies included in our review by Bosetti et al (9) failed to clarify the histology of the oral/pharyngeal cancer cases included, which introduces a potential source of bias. However, over $90 \%$ of oral cancers and $>80 \%$ of pharyngeal cancers are of squamous histology (46), therefore the bias is unlikely to be significant. 
We found that earlier age at menopause is a risk factor for oesophageal SCC, with women entering menopause at $<45$ years having double the risk of those entering menopause at age $>50$ years. Similar, but less striking, results were observed for HNC. HRT was found to reduce the risk of $\mathrm{HNC} /$ oesophageal SCC, but the evidence is inconclusive. We used strict eligibility criteria and only considered studies that controlled for other risk factors, however, there is still the risk of residual bias. Consideration should be given to collecting data on reproductive factors and exposure to HRT, as routine practice, in future epidemiological and clinical studies of these cancers. The concept of oestrogen deficiency as a risk for HNC/oesophageal SCC warrants further investigation in future laboratory and clinical studies.

\section{Acknowledgements}

Mrs. Caroline McCarthy is funded by NIHR (Academic Clinical Fellowship in Oral Medicine). The present review presents independent research funded by the National Institute for Health Research (NIHR). The views expressed are those of the author(s) and not necessarily those of the NHS, the NIHR or the Department of Health.

\section{References}

1. Ferlay J, Soerjomataram I, Dikshit R, Eser S, Mathers C, Rebelo M, Parkin DM, Forman D and Bray F: Cancer incidence and mortality worldwide: Sources, methods and major patterns in GLOBOCAN 2012. Int J Cancer 136: E359-E386, 2015.

2. McCarthy CE, Field JK, Rajlawat BP, Field AE and Marcus MW: Trends and regional variation in the incidence of head and neck cancers in England: 2002 to 2011. Int J Oncol 47: 204-210, 2015.

3. Statistics OfN. Cancer Registration Statistics, England, 2013. 2015.

4. Langevin SM, Grandis JR and Taioli E: Female hormonal and reproductive factors and head and neck squamous cell carcinoma risk. Cancer Lett 310: 216-221, 2011.

5. Bae JM: Modifiable risk factors of lung cancer in 'never-smoker' women. Epidemiol Health 37: e2015047, 2015.

6. Wang BJ, Zhang B, Yan SS, Li ZC, Jiang T, Hua CJ, Lu L, Liu XZ, Zhang DH1, Zhang RS, et al: Hormonal and reproductive factors and risk of esophageal cancer in women: A meta-analysis. Dis Esophagus 29: 448-454, 2016.

7. Green J, Roddam A, Pirie K, Kirichek O, Reeves G and Beral V; Million Women Study collaborators: Reproductive factors and risk of oesophageal and gastric cancer in the Million Women Study cohort. Br J Cancer 106: 210-216, 2012.

8. Freedman ND, Lacey JV Jr, Hollenbeck AR, Leitzmann MF, Schatzkin A and Abnet CC: The association of menstrual and reproductive factors with upper gastrointestinal tract cancers in the NIH-AARP cohort. Cancer 116: 1572-1581, 2010.

9. Bosetti C, Negri E, Franceschi S, Conti E, Levi F, Tomei F and La Vecchia C: Risk factors for oral and pharyngeal cancer in women: A study from Italy and Switzerland. Br J Cancer 82: 204-207, 2000

10. Gallus S, Bosetti C, Franceschi S, Levi F, Simonato L, Negri E and La Vecchia C: Oesophageal cancer in women: Tobacco, alcohol, nutritional and hormonal factors. Br J Cancer 85: 341-345, 2001

11. Yu H, Liu G, Zhao P and Zhu L: Hormonal and reproductive factors and risk of esophageal cancer in Chinese postmenopausal women: A case-control study. Asian Pac J Cancer Prev 12: 1953-1956, 2011

12. Chen ZH, Shao JL, Lin JR, Zhang X and Chen Q: Reproductive factors and oesophageal cancer in Chinese women: A case-control study. BMC Gastroenterol 11: 49, 2011.

13. Lindblad M, García Rodríguez LA, Chandanos E and Lagergren J: Hormone replacement therapy and risks of oesophageal and gastric adenocarcinomas. Br J Cancer 94: 136-141, 2006.
14. Gallus S, Bosetti C, Franceschi S, Levi F, Negri E and La Vecchia C: Laryngeal cancer in women: Tobacco, alcohol, nutritional, and hormonal factors. Cancer Epidemiol Biomarkers Prev 12: 514-517, 2003.

15. Pokoradi AJ, Iversen L and Hannaford PC: Factors associated with age of onset and type of menopause in a cohort of UK women. Am J Obstet Gynecol 205: 34.e1-34.e13, 2011.

16. Faubion SS, Kuhle CL, Shuster LT and Rocca WA: Long-term health consequences of premature or early menopause and considerations for management. Climacteric 18: 483-491, 2015.

17. Monterrosa-Castro A, Blümel JE, Portela-Buelvas K, Mezones-Holguín E, Barón G, Bencosme A, Benítez Z, Bravo LM, Calle A, Chedraui P, et al; Collaborative Group for Research of the Climacteric in Latin America (REDLINC): Type II diabetes mellitus and menopause: A multinational study. Climacteric 16: 663-672, 2013.

18. Stott-Miller M, Chen C, Chuang SC, Lee YC, Boccia S, Brenner H, Cadoni G, Dal Maso L, La Vecchia C, Lazarus P, et al: History of diabetes and risk of head and neck cancer: A pooled analysis from the International Head and Neck Cancer Epidemiology Consortium. Cancer Epidemiol Biomarkers Prev 21: 294-304, 2012.

19. Zhu Y, Yue D, Yuan B, Zhu L and Lu M: Reproductive factors are associated with oesophageal cancer risk: Results from a meta-analysis of observational studies. Eur J Cancer Prev 26: $1-9,2017$.

20. Camargo MC, Goto Y, Zabaleta J, Morgan DR, Correa P and Rabkin CS: Sex hormones, hormonal interventions, and gastric cancer risk: A meta-analysis. Cancer Epidemiol Biomarkers Prev 21: 20-38, 2012.

21. Dietrich K, Demidenko E, Schned A, Zens MS, Heaney J and Karagas MR: Parity, early menopause and the incidence of bladder cancer in women: A case-control study and meta-analysis. Eur J Cancer 47: 592-599, 2011

22. Suba Z: Gender-related hormonal risk factors for oral cancer Pathol Oncol Res 13: 195-202, 2007.

23. Takács D, Koppány F, Mihályi S and Suba Z: Decreased oral cancer risk by moderate alcohol consumption in non-smoker postmenopausal women. Oral Oncol 47: 537-540, 2011.

24. Zong H, Xu H, Geng Z, Ma C, Ming X, Shang M, Li K, He X, $\mathrm{Du} \mathrm{H}$, Zhao J, et al: Reproductive factors in relation to risk of brain tumors in women: An updated meta-analysis of 27 independent studies. Tumour Biol 35: 11579-11586, 2014.

25. Tang B, Lv J, Li Y, Yuan S, Wang Z and He S: Relationship between female hormonal and menstrual factors and pancreatic cancer: A meta-analysis of observational studies. Medicine 94: $\mathrm{e} 177,2015$.

26. Caini S, Gibelli B, Palli D, Saieva C, Ruscica M and Gandini S: Menstrual and reproductive history and use of exogenous sex hormones and risk of thyroid cancer among women: A meta-analysis of prospective studies. Cancer Causes Control 26: 511-518, 2015.

27. Arem H, Park Y, Felix AS, Zervoudakis A, Brinton LA Matthews CE and Gunter MJ: Reproductive and hormonal factors and mortality among women with colorectal cancer in the NIH-AARP Diet and Health Study. Br J Cancer 113: 562-568, 2015.

28. Menon U, Burnell M, Sharma A, Gentry-Maharaj A, Fraser L, Ryan A, Parmar M, Hunter M and Jacobs I; UKCTOCS Group: Decline in use of hormone therapy among postmenopausal women in the United Kingdom. Menopause 14: 462-467, 2007.

29. Rossouw JE, Anderson GL, Prentice RL, LaCroix AZ, Kooperberg C, Stefanick ML, Jackson RD, Beresford SA, Howard BV, Johnson KC, et al; Writing Group for the Women's Health Initiative Investigators: Risks and benefits of estrogen plus progestin in healthy postmenopausal women: Principal results From the Women's Health Initiative randomized controlled trial. JAMA 288: 321-333, 2002.

30. Beral V, Banks E, Reeves G and Bull D; Million Women Study Collaborators: Breast cancer and hormone-replacement therapy in the Million Women Study. Lancet 362: 419-427, 2003.

31. Beral V, Gaitskell K, Hermon C, Moser K, Reeves G and Peto R; Collaborative Group On Epidemiological Studies Of Ovarian Cancer: Menopausal hormone use and ovarian cancer risk: Individual participant meta-analysis of 52 epidemiological studies. Lancet 385: 1835-1842, 2015.

32. Studd J: 'PROFOX' - the post HRT nightmare. Climacteric 14: 217-219, 2011.

33. Ayres J: Premature menopause: Management challenges after the Women's Health Initiative. J Br Menopause Soc 11: 157, 2005. 
34. (NICE) NIfHaCE. Menopause: Diagnosis and management (NG23) 2015. NICE guideline. Published, 12 November 2015. nice.org.uk/guidance/ng23.

35. Murphy N, Strickler HD, Stanczyk FZ, Xue X, WassertheilSmoller S, Rohan TE, Ho GY, Anderson GL, Potter JD and Gunter MJ: A prospective evaluation of endogenous sex hormone levels and colorectal cancer risk in postmenopausal women. J Natl Cancer Inst 107: djv210, 2015.

36. Green J, Czanner G, Reeves G, Watson J, Wise L, Roddam A and Beral V: Menopausal hormone therapy and risk of gastrointestinal cancer: Nested case-control study within a prospective cohort, and meta-analysis. Int J Cancer 130: 2387-2396, 2012.

37. Yao Y, Gu X, Zhu J, Yuan D and Song Y: Hormone replacement therapy in females can decrease the risk of lung cancer: A meta-analysis. PLoS One 8: e71236, 2013.

38. Gandini S, Iodice S, Koomen E, Di Pietro A, Sera F and Caini S Hormonal and reproductive factors in relation to melanoma in women: Current review and meta-analysis. Eur J Cancer 47: 2607-2617, 2011

39. Micheli A, Ciampichini R, Oberaigner W, Ciccolallo L, de Vries E, Izarzugaza I, Zambon P, Gatta G and De Angelis R: EUROCARE Working Group: The advantage of women in cancer survival: An analysis of EUROCARE-4 data. Eur J Cancer 45: 1017-1027, 2009.
40. Utsumi Y, Nakamura T, Nagasue N, Kubota H and Morikawa S: Role of estrogen receptors in the growth of human esophageal carcinoma. Cancer 64: 88-93, 1989.

41. Kalayarasan R, Ananthakrishnan N, Kate V and Basu D: Estrogen and progesterone receptors in esophageal carcinoma. Dis Esophagus 21: 298-303, 2008.

42. Lukits J, Remenár E, Rásó E, Ladányi A, Kásler M and Tímár J: Molecular identification, expression and prognostic role of estrogen- and progesterone receptors in head and neck cancer. Int J Oncol 30: 155-160, 2007.

43. Chandanos E and Lagergren J: The mystery of male dominance in oesophageal cancer and the potential protective role of oestrogen. Eur J Cancer 45: 3149-3155, 2009.

44. Egloff AM, Rothstein ME, Seethala R, Siegfried JM, Grandis JR and Stabile LP: Cross-talk between estrogen receptor and epidermal growth factor receptor in head and neck squamous cell carcinoma. Clin Cancer Res 15: 6529-6540, 2009.

45. Chang YL, Hsu YK, Wu TF, Huang CM, Liou LY, Chiu YW, Hsiao YH, Luo FJ and Yuan TC: Regulation of estrogen receptor $\alpha$ function in oral squamous cell carcinoma cells by FAK signaling. Endocr Relat Cancer 21: 555-565, 2014.

46. Davies L and Welch HG: Epidemiology of head and neck cancer in the United States. Otolaryngol Head Neck Surg 135: 451-457, 2006. 